Original Paper http://ajol.info/index.php/ijbcs http://indexmedicus.afro.who.int

\title{
La flore adventice des cultures cotonnières dans le Sénégal Oriental et en Haute Casamance
}

\author{
Ndongo DIOUF ${ }^{1,2^{*}}$, Mame Samba MBAYE ${ }^{1}$, Moustapha GUEYE $^{3}$, Birane DIENG ${ }^{1}$, \\ César BASSENE ${ }^{4}$ et Kandioura NOBA ${ }^{1}$ \\ ${ }^{I}$ Laboratoire de Botanique Biodiversité, Département de Biologie Végétale, Faculté des sciences et technique, \\ Université cheikh Anta Diop, B.P. 5005 Dakar- Fann, Sénégal. \\ ${ }^{2}$ Centre de recherches zootechniques de Kolda, Institut Sénégalais de Recherche Agricoles, B.P 53 Kolda, \\ Sénégal. \\ ${ }^{3}$ Centre National de Recherches Agronomiques de Bambey, Institut Sénégalais de Recherche Agricoles, B.P 53 \\ Kolda, Sénégal. \\ ${ }^{4}$ UFR des Sciences Agronomiques, de l'Aquaculture et des Technologies Alimentaires, Université Gaston \\ Berger de Saint Louis, B.P.234 Sénégal. \\ *Auteur correspondant ; E-mail : ndongodiouf87@gmail.com; Tel : +221 774566580
}

\section{RESUME}

Au Sénégal, les adventices sont l'une des principales contraintes à la production cotonnière. La présente étude a pour objectif de caractériser la flore adventice des cultures cotonnières au Sénégal. Des relevés phytosociologiques ont été réalisés durant les campagnes agricoles 2016, 2017 et 2018 dans les stations de recherche de l'ISRA (Institut Sénégalais de Recherche Agricole), dans les AMEX (Antenne Multilocale d'Expérimentation) de la SODEFITEX (Société de Développement de Fibres Textiles du Sénégal) et en milieu paysan. Grâce aux inventaires phytosociologiques par la technique du «tour de champs » qui consiste à parcourir toute la parcelle dans toutes ces directions pour répertorier toutes les espèces même les plus rares. Les résultats de cette étude montrent que cette flore est riche de 204 espèces réparties en 118 genres et 35 familles. Ces dernières sont dominées par les Fabaceae (22,1\%), Poaceae (20,1\%), les Malvaceae $(9,3 \%)$ et les Cyperaceae $(7,8 \%)$. Les Dicotylédones sont dominantes avec 68,6\% des espèces. Cette flore est nettement dominée par les thérophytes avec $81,4 \%$. Sur le plan de la répartition géographique, les espèces à affinité pantropicales et d'origine Africaines sont dominantes et elles regroupent près de $74,1 \%$ des espèces répertoriées. Le bassin cotonnier sénégalais est aussi caractérisé par une homogénéité floristique. Ce travail a permis de caractériser la flore adventice des cultures cotonnières dans le Sénégal oriental et en haute Casamance.

() 2019 International Formulae Group. All rights reserved

Mots clés : Flore, adventice, Coton, Bassin cotonnier du Sénégal, ISRA

\section{Weed flora of cotton crops in Eastern Senegal and Upper Casamance}

\section{ABSTRACT}

In Senegal, among the factors limiting cotton production, weeds competition had been identified as a major obstacle in increasing the fibber yield. This study was carried out in Eastern Senegal and Upper 
Casamance to characterize the weed flora of cotton. It aimed to establish the taxonomical, life forms and chorological spectra of weed species. Therefore, floristic surveys were carried out during 2016, 2017 and 2018 crop years in cotton fields and in stations. The "field tower" technique, which consists of taking stock of all species in a defined area, has been adopted. The results revealed that flora consisted of 204 species distributed in 118 genera and 35 families. The families with the highest species richness were Fabaceae (22.1\%), Poaceae (20.1\%), Malvaceae $(9.3 \%)$ and Cyperaceae $(7.8 \%)$ which account for sixty percent of recorded species. Dicotyledon was the most important form with 68.6 percent of recorded species. Spectrum analysis indicated that the flora is largely dominated by therophytes, which includes $81.4 \%$ of the recorded species. The study of the biogeographical distribution showed that african and pantropical species predominate with 74.1 percent of the flora. The Senegalese cotton basin is also characterized by a homogeneity in the floristic composition despite some variations

(C) 2019 International Formulae Group. All rights reserved

Keywords: Weeds, flora, Cotton Basin of Senegal, Senegal.

\section{INTRODUCTION}

Au Sénégal, notamment dans la partie sud-Est du pays, la culture du coton est une source importante de revenus pour un grand nombre de producteurs agricoles. Dans cette zone, le coton constitue la deuxième culture de rente après l'arachide et contribue pour $2 \%$ du PIB et engage 40000 à 80000 producteurs (Diaw, 2010).

La culture du coton est soumise à de nombreuses contraintes biotiques et abiotiques contribuant à la baisse des rendements. En effet, les pertes de rendement notées dans les cultures cotonnières au Sénégal sont dues entre autres au manque d'intrants agricoles; le manque d'encadrement technique des petits producteurs ; aux maladies ; aux ravageurs tels que Helicoverpa armigera ; ainsi qu'aux adventices.

Les adventices constituent une des contraintes majeures dans la culture cotonnière. Elles causent une perte de rendement pouvant dépasser $25 \%$ en zone tropicale (Boudjedjou, 2010). Ce sont des plantes qui peuvent être envahissantes de par leur capacité à produire une grande quantité de graines. Ces plantes entrent en compétition pour l'eau, les nutriments avec la plante cultivée et constituent des réservoirs pour un certain nombre de virus communs. Dans le bassin cotonnier du Sénégal, l'augmentation des surfaces des cultures cotonnières a pris une importance telle que la gestion des adventices est devenue particulièrement préoccupante. Face à tous ces problèmes que posent les adventices, il est important qu'une stratégie de lutte efficace de l'enherbement soit mise en œuvre. Or, dans la mise en œuvre de cette stratégie de lutte, la connaissance de la flore est essentielle. Toutefois, dans le bassin cotonnier du Sénégal, peu de données sur les adventices sont disponibles. Le présent travail a pour objectif d'identifier la flore adventice des cultures cotonnières dans le Sénégal oriental et en haute Casamance.

\section{MATERIEL ET METHODES}

Ce travail se propose d'analyser la composition de la flore adventice, d'établir le spectre biologique, la distribution géographique et de faire une étude comparative des trois localités (Zone sèche, Zone intermédiaire et Zone humide) en fonction de la flore adventices.

\section{Zone d'étude}

La région concernée par le présent travail est le bassin cotonnier sénégalais qui regroupe 4 régions administratives à savoir Tambacounda, Kolda, Kédougou et Kaolack (Figure 1). 
Le bassin cotonnier, couvre près de $46 \%$ du territoire national et occupe $27 \%$ de la population sénégalaise (Ndour et al., 2017).

Conformément au gradient de pluviométrie décrit par Ndong (1995), le bassin cotonnier Sénégalais est caractérisé par 3 zones climatiques différentes :

○ Zone sèche au Nord -Ouest du bassin avec une pluviométrie moyenne annuelle de 600 à $800 \mathrm{~mm}$

- Zone intermédiaire au Nord -Est du bassin avec une pluviométrie moyenne annuelle de 800 à $900 \mathrm{~mm}$

Zone humide qui couvre les parties Est et Ouest du Parc National de Niokolo koba ; partie la plus pluvieuse du pays avec une pluviométrie moyenne annuelle de 900 à 1200 mm.

\section{Méthode}

L'inventaire des adventices a été effectué au cours du cycle cultural du cotonnier en 2016, 2017 et 2018. La technique des relevés floristiques est celle du tour de champs, qui permet de recenser les différentes espèces de la parcelle de façon exhaustive. Elle consiste à parcourir la parcelle dans différentes directions jusqu'à ce que la découverte d'une espèce nouvelle nécessite un parcourt important (Maillet, 1981; Le Bourgeois, 1993). Cette méthode à l'avantage de prendre en compte l'hétérogénéité de la parcelle. De plus selon Maillet (1981) cette technique permet de tenir compte d'espèces rares, mais de grande importance d'un point de vue agronomique notamment les espèces à extension rapide où les espèces indicatrices de certaines caractéristiques du milieu.

L'identification des espèces recensées a été faite à partir des flores de Berhaut (1967), de Lebrun et Stork (1991-1997) de l'herbier du Département de Biologie Végétale de la Faculté des Sciences et
Techniques de l'Université Cheikh Anta Diop de Dakar.

A la suite de l'inventaire floristique, différentes notions ont été déterminées. Il s'agit de :

Spectre biologique: Pour les types biologiques, le modèle de classification adopté est celui Raunkiaer (1934). Les pourcentages des espèces appartenant au même type biologique permettent de déterminer les spectres biologiques.

Répartition géographique: Pour la répartition phytogéographique, les informations sont obtenues grâce à la flore de Hutchinson, les travaux de Thiombiano et al. (2012).

Coefficient de similitude : Le degré de ressemblance entre les floreS des trois localités (zone sèche, intermédiaire et humide) a été déterminé par la méthode des coefficients de similitude (Cs). La formule utilisée, telle que présentée ci-après, est celle de Sørensen (1948).

$$
\mathrm{CS}=\frac{2 c}{A+B}
$$

A représente le nombre d'espèces appartenant à une liste (A), dressée à l'issue de l'inventaire effectué dans une localité donnée ;

$B$ représente le nombre d'espèces appartenant à une liste que l'on veut comparer à la première liste ; (C) est le nombre d'espèces communes à $(\mathrm{A})$ et $(\mathrm{B})$. Cs varie donc entre 0 et $100 \%$. Lorsque Cs est supérieur ou égal à $50 \%$, cela signifie que les deux listes comparées sont très proches l'une de l'autre au point d'être assimilées à des milieux identiques ; en d'autres termes, les deux milieux concernés peuvent être considérés comme floristiquement homogènes (Gounot, 1969 ; Guinochet, 1973).

Ce coefficient permet de vérifier l'homogénéité des sites de relevés pris deux à deux au regard de leur composition floristique. 


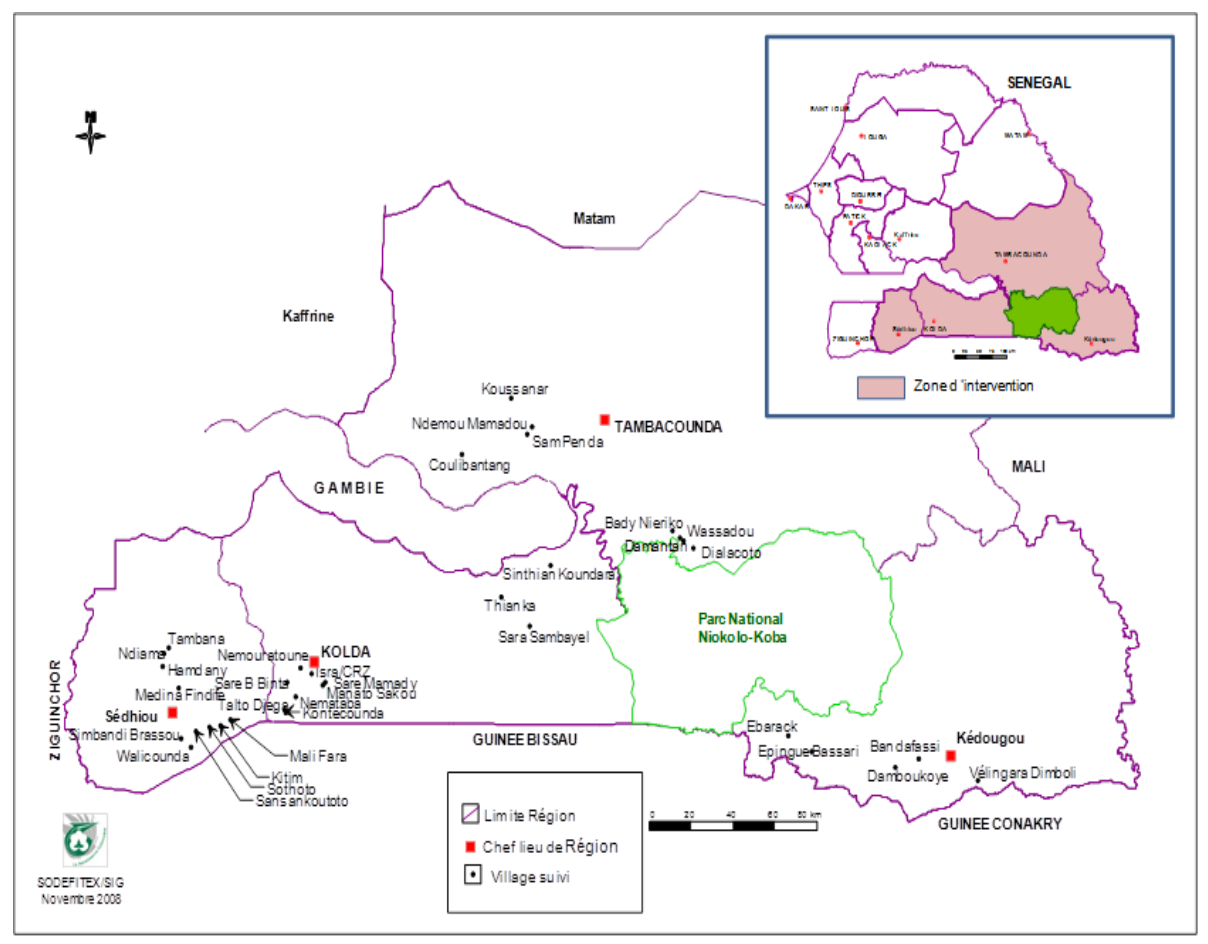

Source : SODEFITEX, 2008.

Figure 1 : carte du bassin cotonnier du Sénégal.

\section{RESULTATS}

\section{Analyse de la flore}

\section{Structure globale de la flore}

$\mathrm{La}$ liste globale des espèces rencontrées est consignée dans le Tableau 1. Chaque espèce est représentée avec son type biologique (TB) et sa répartition géographique (RG).

Lors de cette étude, 204 espèces d'adventices ont été recensées dans les parcelles cotonnières. Ces espèces sont réparties dans 118 genres et 35 familles. Dans cette flore, les dicotylédones dominent avec respectivement $84,8 \%$ des familles, $70,4 \%$ des genres et $69,5 \%$ des espèces (Tableaux 1 et 2 ).

Le Tableau 3 donne par ordre d'importance le nombre d'espèces par famille et leur contribution à l'effectif total. Quatre familles dominent nettement la flore adventice dans ce bassin cotonnier : Il s'agit des Fabaceae avec (22,1\%), Poaceae avec $(20,1 \%)$, Malvaceae avec $(9,3 \%)$ et
Cyperaceae avec (7,8\%). Ces familles totalisent à elles seules 117 espèces soit 59,4 $\%$ de l'effectif global (Tableau 3). Il ressort également de ces résultats que certaines familles présentent des proportions non négligeables. Parmi celles-ci, on peut citer les Convolvulaceae, les Asteraceae et les Rubiaceae avec chacune $4,9 \%$. Les familles restantes contribuent pour $25,9 \%$ des espèces rencontrées et ne sont représentées que par 1 à 6 espèces par famille.

\section{Spectre biologique}

Les répartitions des types biologiques des adventices de la zone cotonnière du Sénégal sont consignées dans la figure 2. La classification par ordre d'importance des types biologiques montre que les thérophytes dominent largement cette flore avec 166 espèces soit $81,4 \%$ de la flore adventice. Les autres types biologiques sont faiblement représentés et ne dépassent pas les $6 \%$ des adventices listées. 


\section{Spectre chorologique}

La répartition phytogéographique des espèces d'adventices recensées dans les zones cotonnières du Sénégal montre une très grande diversité (Figure 3).

Les espèces d'affinité pantropicales $(41,7 \%)$ et africaines $(32,4 \%)$ constituent la majorité avec $74,1 \%$ de l'ensemble des espèces répertoriées.

Le reste, $(25,9 \%)$, des affinités phytogéographiques est représenté par des espèces d'origine Afro-asiatiques et australiennes, Cosmopolites, Afro-asiatiques, Afro-malgaches et asiatiques, américaines,
Afro-Américaines et Asiatiques, Afromalgaches asiatiques et australiennes et Afroaméricaines et australiennes.

\section{Influence du site sur la diversité floristique}

Le nombre d'espèce par Sites: Zone humide, Zone intermédiaire et Zone Sèche est, respectivement indiqué, 186 espèces, 103 espèces et 83 espèces. Les indices de similitude sont tous supérieur à $60 \%$ (Tableau 4). Sur la base de ces résultats, ces trois sites sont similaires du point de vue de la diversité spécifique des adventices.

Tableau 1 : Liste des espèces recensées avec des indications sur leur type biologique (T.B) et leur répartition géographique (R.G).

\begin{tabular}{|c|c|c|c|}
\hline Famille & Espèce & RG & \\
\hline \multirow{4}{*}{ ACANTHACEAE } & Blepharis maderaspatensis (L.) & $\mathrm{T}$ & Af \\
\hline & B.Heyne ex Roth & & \\
\hline & Monechma ciliatum (Jacq.) & $\mathrm{T}$ & Af \\
\hline & Milne-Redh. & & \\
\hline \multirow{2}{*}{ AIZOACEAE } & Sesuvium portulacastrum (L.) L. & $\mathrm{T}$ & $\mathrm{Pt}$ \\
\hline & Trianthema portulacastrum $\mathrm{L}$. & $\mathrm{T}$ & $\mathrm{Pt}$ \\
\hline \multirow{5}{*}{ AMARANTHACEAE } & Amaranthus cruentus (L.) Thell. & $\mathrm{T}$ & Af \\
\hline & Amaranthus spinosus L. & $\mathrm{T}$ & Cosm \\
\hline & Celosia laxa Schumach. \& & $\mathrm{T}$ & $\mathrm{Tt}$ \\
\hline & Thonn. & & \\
\hline & Celosia trigyna $\mathrm{L}$. & $\mathrm{T}$ & As \\
\hline \multirow{2}{*}{ ARACEAE } & Stylochiton hypogaeus Lepr. & G & Af \\
\hline & Stylochiton warnakaei Sw. & $\mathrm{T}$ & Masu \\
\hline \multirow{2}{*}{ ASPARAGACEAE } & Asparagus flagellaris (Kunth) & $\mathrm{P}$ & Af \\
\hline & Baker & & \\
\hline \multirow{10}{*}{ ASTERACEAE } & Acanthospermum hispidium DC. & $\mathrm{P}$ & Af \\
\hline & Achyrantes aspera $\mathrm{L}$. & $\mathrm{T}$ & Cosm \\
\hline & Ageratum conyzoides L. & $\mathrm{T}$ & $\mathrm{Pt}$ \\
\hline & Blumea aurita (DC.) C.D Adams & $\mathrm{T}$ & $\mathrm{Pt}$ \\
\hline & Blainvillea gayana Cass. & $\mathrm{T}$ & Af \\
\hline & $\begin{array}{l}\text { Conysa sumatruens (Retz.) E. } \\
\text { Walker }\end{array}$ & $\mathrm{T}$ & Mas \\
\hline & Launaea intybacea (Jacq.) & $\mathrm{T}$ & $\mathrm{Pt}$ \\
\hline & Beauverd & & \\
\hline & Mollugo nudicaulis Lam. & $\mathrm{T}$ & AmAs \\
\hline & Synedrella nodiflora Gaertn. & $\mathrm{T}$ & $\mathrm{Pt}$ \\
\hline
\end{tabular}




\begin{tabular}{|c|c|c|c|}
\hline & $\begin{array}{l}\text { Vernonia galamensis (Cass.) } \\
\text { Less. }\end{array}$ & $\mathrm{T}$ & Af \\
\hline CAPPARACEAE & Cleome viscosa $\mathrm{L}$. & $\mathrm{T}$ & $\mathrm{Pt}$ \\
\hline CARYOPHYLLACEAE & $\begin{array}{l}\text { Polycarpaea corymbosa (L.) } \\
\text { Lam. }\end{array}$ & $\mathrm{P}$ & Af \\
\hline COLCHICACEAE & Gloriosa simplex $\mathrm{L}$. & $\mathrm{T}$ & $\mathrm{Pt}$ \\
\hline \multirow{4}{*}{ COMBRETACEAE } & Combretum geitinophyllum Diels & $\mathrm{T}$ & Cosm \\
\hline & $\begin{array}{l}\text { Combretum glutinosum Perr. ex } \\
\text { DC. }\end{array}$ & $\mathrm{P}$ & Af \\
\hline & Guiera senegalensis J.F.Gmel. & $\mathrm{T}$ & Af \\
\hline & $\begin{array}{l}\text { Terminalia macroptera Guill. \& } \\
\text { Perr. }\end{array}$ & $\mathrm{P}$ & Af \\
\hline \multirow{3}{*}{ COMMELINACEAE } & Commelina benghalensis L. & $\mathrm{T}$ & $\mathrm{Tp}$ \\
\hline & Commelina forskalaei Vahl. & $\mathrm{T}$ & As \\
\hline & $\begin{array}{l}\text { Commelina gambiae (C.B. } \\
\text { Clarke) }\end{array}$ & $\mathrm{T}$ & Mas \\
\hline \multirow{10}{*}{ CONVOLVULACEAE } & $\begin{array}{l}\text { Ipomoea acanthocarpa (Choisy) } \\
\text { Asch }\end{array}$ & $\mathrm{T}$ & Af \\
\hline & Ipomoea coptica (L.) Roth. & $\mathrm{C}$ & Af \\
\hline & Ipomoea eriocarpa $\mathrm{R} . \mathrm{Br}$. & $\mathrm{T}$ & Asu \\
\hline & Ipomoea heterotricha Didr. & $\mathrm{T}$ & $\mathrm{Am}$ \\
\hline & Ipomoea pes-tigridis $\mathrm{L}$. & $\mathrm{T}$ & $\mathrm{Pt}$ \\
\hline & Ipomoea vagans Bak. & $\mathrm{T}$ & Af \\
\hline & $\begin{array}{l}\text { Jacquemontia tamnifolia (L.) } \\
\text { Griseb. }\end{array}$ & $\mathrm{T}$ & Af \\
\hline & Merremia pentaphylla (L.)Halier. & $\mathrm{T}$ & $\mathrm{Pt}$ \\
\hline & $\begin{array}{l}\text { Merremia pinnata (Hochst.) } \\
\text { Hallier. }\end{array}$ & $\mathrm{T}$ & $\mathrm{Pt}$ \\
\hline & $\begin{array}{l}\text { Merremia tridentata }(\mathrm{L} .) \text { Hallier } \\
\text { f. }\end{array}$ & $\mathrm{T}$ & Af \\
\hline \multirow{6}{*}{ CUCURBITACEAE } & Colosyntus vulgaris (L.) O.Kze. & $\mathrm{T}$ & Af \\
\hline & Cucumis melo $\mathrm{L}$. & $\mathrm{T}$ & As \\
\hline & Cucurbita maxima Duch & $\mathrm{T}$ & Af \\
\hline & $\begin{array}{l}\text { Lagenaria siceraria (Molina) } \\
\text { Standl. }\end{array}$ & $\mathrm{G}$ & Af \\
\hline & Luffa acutangula (L.) M.Roem. & $\mathrm{T}$ & Af \\
\hline & Luffa cylindrica (L.) M.Roem. & $\mathrm{T}$ & $\mathrm{Pt}$ \\
\hline \multirow{5}{*}{ CYPERACEAE } & $\begin{array}{l}\text { Bulbostylis barbata (Rottb.) } \\
\text { C.B.Clarke }\end{array}$ & $\mathrm{T}$ & Af \\
\hline & Bulbostylis hispidula (Vahl) & $\mathrm{T}$ & $\mathrm{Pt}$ \\
\hline & $\begin{array}{l}\text { R.W.Haines } \\
\text { Cyperus amabilis Vahl }\end{array}$ & $\mathrm{G}$ & Cosm \\
\hline & Cyperus cuspidatus Kunth & $\mathrm{T}$ & $\mathrm{Pt}$ \\
\hline & Cyperus difformis $\mathrm{L}$. & $\mathrm{T}$ & $\mathrm{Pt}$ \\
\hline
\end{tabular}




\begin{tabular}{|c|c|c|c|c|}
\hline & Cyperus esculentus L. [cult.] & $\mathrm{T}$ & $\mathrm{Pt}$ \\
\hline & & Cyperus iria $\mathrm{L}$. & $\mathrm{T}$ & $\mathrm{Pt}$ \\
\hline & & Cyperus rotundus $\mathrm{L}$. & $\mathrm{G}$ & $\mathrm{Pt}$ \\
\hline & & Cyperus sphacelatus Rottb. & $\mathrm{T}$ & $\mathrm{Pt}$ \\
\hline & & Fimbristylis exilis (Kunth) & $\mathrm{T}$ & Af \\
\hline & & Roem.\&Schult. & & \\
\hline & & Kyllinga erecta Schumach. & $\mathrm{T}$ & Af \\
\hline & & $\begin{array}{l}\text { Kyllinga squamulata Thon.et } \\
\text { Vahl. }\end{array}$ & $\mathrm{T}$ & Am \\
\hline & & Kyllinga pumilla Mich. & $\mathrm{H}$ & Af \\
\hline & & Mariscus cylindristachyus Steud. & $\mathrm{T}$ & $\mathrm{Pt}$ \\
\hline & & Mariscus hamilosus (M. Bieb) & $\mathrm{T}$ & $\mathrm{Pt}$ \\
\hline & & Mariscus squarrosus (L.) & $\mathrm{T}$ & $\mathrm{Ct}$ \\
\hline & & C.B.Clarke & & \\
\hline & & Pycreus lanceolatus (Poir.) & $\mathrm{G}$ & Masu \\
\hline & & C.B.Clarke & & \\
\hline \multirow{5}{*}{\multicolumn{2}{|c|}{ EUPHORBIACEAE }} & Acalypha ciliata Forsk. & $\mathrm{T}$ & $\mathrm{Pt}$ \\
\hline & & Chrozophora senegalensis & $\mathrm{C}$ & Af \\
\hline & & (Lam.) A.Juss. ex Spreng. & & \\
\hline & & Euphorbia convolvuloides & $\mathrm{T}$ & Asu \\
\hline & & $\begin{array}{l}\text { Hochst. ex Benth. } \\
\text { Euphorbia hirta L. }\end{array}$ & $\mathrm{T}$ & AS \\
\hline \multirow{17}{*}{ FABACEAE } & \multirow{10}{*}{ CAESALPINOIDAEA } & Cassia absus L. & $\mathrm{T}$ & Af \\
\hline & & Cassia mimosoides L. & $\mathrm{T}$ & $\mathrm{Pt}$ \\
\hline & & Cassia nigricans Vahl & $\mathrm{T}$ & $\mathrm{Pt}$ \\
\hline & & Cassia obtusifolia L. & $\mathrm{T}$ & $\mathrm{Pt}$ \\
\hline & & Cassia occidentalis L. & $\mathrm{T}$ & $\mathrm{Pt}$ \\
\hline & & Cassia rotundifolia Pers. & $\mathrm{T}$ & $\mathrm{Pt}$ \\
\hline & & Piliostigma reticulatum (DC.) & $\mathrm{T}$ & $\mathrm{Pt}$ \\
\hline & & Hochst. & & \\
\hline & & $\begin{array}{l}\text { Piliostigma thonningii } \\
\text { (Schumach.) Milne-Redh. }\end{array}$ & $\mathrm{P}$ & Af \\
\hline & & Sesbania pachycarpa DC. & $\mathrm{T}$ & Asu \\
\hline & \multirow{7}{*}{ FABOIDAEA } & $\begin{array}{l}\text { Aeschynomene tambacoundensis } \\
\text { Berhaut }\end{array}$ & $\mathrm{T}$ & Af \\
\hline & & $\begin{array}{l}\text { Alysicarpus ovalifolius } \\
\text { (Schumach.) Léonard }\end{array}$ & $\mathrm{T}$ & $\mathrm{Pt}$ \\
\hline & & Arachis hypogaea L. & $\mathrm{T}$ & Am \\
\hline & & $\begin{array}{l}\text { Atylosia scarabaeoides (L.) } \\
\text { Benth. }\end{array}$ & $\mathrm{T}$ & Af \\
\hline & & Calopogonium mucunoides Desv. & $\mathrm{T}$ & $\mathrm{Pt}$ \\
\hline & & Crotalaria deightonii Hepper & $\mathrm{T}$ & $\mathrm{Pt}$ \\
\hline & & $\begin{array}{l}\text { Crotalaria goreensis Guill. \& } \\
\text { Perr. }\end{array}$ & $\mathrm{T}$ & Af \\
\hline
\end{tabular}


Crotalaria juncea $\mathrm{L}$.

Crotalaria retusa $\mathrm{L}$.

Desmodium gangeticum (L.) DC

Desmodium ospriostreblum

Chiov.

Indigofera dendroides Jacq.

Indigofera hirsuta $\mathrm{L}$.

Indigofera macrocalyx Guill. \&

Perr.

Indigofera nummulariifolia $(\mathrm{L}.) \quad \mathrm{T}$

Livera ex Alston

Indigofera pilosa Poir.

Indigofera secundiflora Poir.

Indigofera senegalensis Lam.

Indigofera stenophylla Guill. \&

Perr.

Indigofera tinctoria $\mathrm{L}$.

Mucuna maderaspatana (L.)

Roem.

Rhynchosia minima (L.) DC.

Stylosanthes fruticosa (Retz.)

Alston

Tephrosia bracteolata Guill. et $\quad \mathrm{T}$

Perr.

Tephrosia elegans Schumach

Tephrosia linearis (Willd) Pers

Tephrosia pedicellata Back.

Tephrosia platycarpa Guill. \&

Perr.

Tephrosia purpurea (L.) Pers.

Vigna racemosa (G.Don) Hutch. T

\& Dalziel

Vigna radiata (L.) R.Wilczek. T

Vigna unguculata (L.) Walp.

Zornia glochidiata Rchb. ex DC.

Acacia seyal Delile

Dichrostachys cinerea (L.) Wight $\mathrm{T}$

\& Arn.

\begin{tabular}{lll} 
Icacina senegalensis A. Juss & $\mathrm{T}$ & $\mathrm{Af}$ \\
\hline Hyptis spicigera Lam. & $\mathrm{T}$ & $\mathrm{Pt}$ \\
Hyptis suaveolens Poit. & $\mathrm{T}$ & $\mathrm{AmAs}$ \\
Leucas martinicensis (Jacq.) & $\mathrm{T}$ & Cosm \\
R.Br. & &
\end{tabular}

Af

$\mathrm{Pt}$

$\mathrm{Pt}$

$\mathrm{Pt}$

T AmAs

$\mathrm{H}$ AmAs

G Af

Af

T Af

$T \quad$ Amu

C Af

T Mas

$\mathrm{T}$ Af

T Af

T Mas

T Pt

G Af

T Cosm

$T$ Af

T Af

$\mathrm{T}$ Af

T Pt

T Af

T Pt

T Pt

T $\mathrm{Pt}$

T Mas

$\mathrm{R}$ Br.
Indigofera pulchra Willd.

T Af

MIMOSOIDAEA 


\begin{tabular}{|c|c|c|c|}
\hline LILIACEAE & Scilla sudanica A.Chev. & $\mathrm{He}$ & $\mathrm{Pt}$ \\
\hline LOGANIACEAE & Strychnos spinosa Lam. & G & Af \\
\hline \multirow{13}{*}{ MALVACEAE } & Abelmoschus esculentus (L.) & $\mathrm{C}$ & Cosm \\
\hline & Moench & & \\
\hline & Hibiscus asper Hook.f. & $\mathrm{G}$ & Af \\
\hline & Hibiscus cannabinus L. & $P$ & Af \\
\hline & Hibiscus diversifolius Jacq. & $\mathrm{T}$ & $\mathrm{Pt}$ \\
\hline & Hibiscus sabdariffa $\mathrm{L}$. & $\mathrm{T}$ & $\mathrm{Pt}$ \\
\hline & Sida alba $\mathrm{L}$. & $\mathrm{T}$ & Et \\
\hline & Sida linifolia Juss. ex Cav. & $\mathrm{C}$ & Af \\
\hline & Sida ovata Forsk. & $\mathrm{T}$ & $\mathrm{Pt}$ \\
\hline & Sida rhombifolia $\mathrm{L}$. & $\mathrm{T}$ & $\mathrm{Pt}$ \\
\hline & Sida stipulata Cav. & $\mathrm{T}$ & $\mathrm{Pt}$ \\
\hline & Sida urens L. & $\mathrm{T}$ & $\mathrm{Pt}$ \\
\hline & $\begin{array}{l}\text { Wissadula amplissima (L.) R. E. } \\
\text { Fr. }\end{array}$ & $\mathrm{T}$ & Af \\
\hline \multirow{6}{*}{ TILIOIDEAE } & Corchorus aestuans $\mathrm{L}$. & $\mathrm{T}$ & $\mathrm{Pt}$ \\
\hline & Corchorus olitorius L. & $\mathrm{T}$ & $\mathrm{Pt}$ \\
\hline & Corchorus tridens $\mathrm{L}$. & $\mathrm{T}$ & $\mathrm{Pt}$ \\
\hline & Triumfetta pentandra A. Rich. & $\mathrm{T}$ & $\mathrm{Pt}$ \\
\hline & Urena lobata $\mathrm{L}$. & $\mathrm{T}$ & $\mathrm{Pt}$ \\
\hline & Walteria indica $\mathrm{L}$. & $\mathrm{C}$ & $\mathrm{Pt}$ \\
\hline \multirow{2}{*}{ NYCTAGINACEAE } & Boerhavia diffusa $\mathrm{L}$. & $\mathrm{T}$ & $\mathrm{Pt}$ \\
\hline & Boerhavia erecta $\mathrm{L}$. & $\mathrm{T}$ & $\mathrm{Pt}$ \\
\hline ONAGRACEAE & Ludwigia abyssinica A.Rich. & $\mathrm{H}$ & Af \\
\hline \multirow{2}{*}{ PEDALIACEAE } & Ocimum canum Sims & $\mathrm{T}$ & $\mathrm{Pt}$ \\
\hline & Sesamum indicum $\mathrm{L}$. & $\mathrm{T}$ & Asu \\
\hline PHYLLANTHACEAE & $\begin{array}{l}\text { Phyllanthus amarus Schum. \& } \\
\text { Thorm. }\end{array}$ & $\mathrm{T}$ & Af \\
\hline \multirow{8}{*}{ POACEAE } & $\begin{array}{l}\text { Acroceras zizanioides (Kunth) } \\
\text { Dandy }\end{array}$ & $\mathrm{H}$ & AmAs \\
\hline & Andropogon gayanus Kunth & $\mathrm{H}$ & Af \\
\hline & Andropogon pseudapricus Stapf & $\mathrm{T}$ & AmAs \\
\hline & $\begin{array}{l}\text { Brachiaria deflexa (Schumach.) } \\
\text { Hubb. }\end{array}$ & $\mathrm{T}$ & Mas \\
\hline & $\begin{array}{l}\text { Brachiaria lata (Schumach.) } \\
\text { Hubb. }\end{array}$ & $\mathrm{T}$ & Mas \\
\hline & Brachiaria ramosa $($ L.) Stapf & $\mathrm{T}$ & $\mathrm{P}$ \\
\hline & $\begin{array}{l}\text { Brachiaria stigmatisata }(\mathrm{Mez}) \\
\text { Stapf. }\end{array}$ & $\mathrm{T}$ & Asu \\
\hline & $\begin{array}{l}\text { Brachiaria villosa (Lam.) A. } \\
\text { Camus }\end{array}$ & $\mathrm{T}$ & Af \\
\hline
\end{tabular}




\begin{tabular}{|c|c|c|}
\hline $\begin{array}{l}\text { Brachiaria xantholeuca (Hack) } \\
\text { Stapf }\end{array}$ & $\mathrm{T}$ & $\mathrm{Pt}$ \\
\hline Cenchrus biflorus Roxb. & $\mathrm{T}$ & As \\
\hline Chloris pilosa Schum. & $\mathrm{T}$ & Af \\
\hline Ctenium elegans Kunth & $\mathrm{T}$ & Af \\
\hline Cynodon dactylon (L.) Pers. & $\mathrm{T}$ & Cosm \\
\hline $\begin{array}{l}\text { Dactyloctenium aegyptium } \\
\text { Beauv. }\end{array}$ & $\mathrm{G}$ & $\mathrm{Pt}$ \\
\hline Digitaria horizontalis Willd. & $\mathrm{T}$ & Af \\
\hline Echinochloa colona (L.) Link & $\mathrm{T}$ & $\mathrm{Pt}$ \\
\hline Eleusine indica (L.) Gaertn. & $\mathrm{T}$ & $\mathrm{Pt}$ \\
\hline Eragrostis aspera (Jacqu.) Ness & $\mathrm{T}$ & Mas \\
\hline Eragrostis ciliaris (L.) R. Br. & $\mathrm{T}$ & $\mathrm{Pt}$ \\
\hline Eragrostis pilosa (L.) P. Beauv. & $\mathrm{T}$ & Cosm \\
\hline $\begin{array}{l}\text { Eragrostis tremula Hochst. ex } \\
\text { Steud. }\end{array}$ & $\mathrm{T}$ & $\mathrm{Pt}$ \\
\hline Eragrostis tenella Roem. et Sch. & $\mathrm{T}$ & $\mathrm{Pt}$ \\
\hline Eragrostis turgida De Wild. & $\mathrm{T}$ & $\mathrm{Pt}$ \\
\hline $\begin{array}{l}\text { Euclasta condylotricha (Hochst. } \\
\text { ex Steud.) Stapf }\end{array}$ & $\mathrm{T}$ & Af \\
\hline $\begin{array}{l}\text { Hackelochloa granularis (L.) } \\
\text { Kuntze }\end{array}$ & $\mathrm{T}$ & Mas \\
\hline $\begin{array}{l}\text { Loudetiopsis pobeguinii (Jacq.- } \\
\text { Fél.) Clayton }\end{array}$ & $\mathrm{T}$ & $\mathrm{Pt}$ \\
\hline $\begin{array}{l}\text { Oplismenus burmannii (Retz.) } \\
\text { P.Beauv. }\end{array}$ & $\mathrm{T}$ & Mas \\
\hline Oryza sativa $\mathrm{L}$. & $\mathrm{T}$ & $\mathrm{Pt}$ \\
\hline Oryza barthii A.Chev. & $\mathrm{T}$ & Af \\
\hline Paspalum geminatum Stapf & $\mathrm{T}$ & $\mathrm{Am}$ \\
\hline Paspalum scrobiculatum $\mathrm{L}$. & $\mathrm{T}$ & $\mathrm{Am}$ \\
\hline Pennisetum glaucum (L.) R.Br. & $\mathrm{H}$ & $\mathrm{Pt}$ \\
\hline Pennisetum pedicellatum Trin. & $\mathrm{T}$ & AmAs \\
\hline $\begin{array}{l}\text { Pennisetum polystachion (L.) } \\
\text { Schult. }\end{array}$ & $\mathrm{T}$ & $\mathrm{Pt}$ \\
\hline $\begin{array}{l}\text { Pennisetum violaceum } \\
\text { (Lam.)Rich }\end{array}$ & $\mathrm{T}$ & $\mathrm{Pt}$ \\
\hline $\begin{array}{l}\text { Rottboellia cochinchinensis } \\
\text { (Lour.) Clayton }\end{array}$ & $\mathrm{T}$ & $\mathrm{Pt}$ \\
\hline $\begin{array}{l}\text { Schizachyrium sanguineum } \\
\text { (Retz.) Alston }\end{array}$ & $\mathrm{T}$ & Mas \\
\hline Schoenefeldia gracilis Kunth & $\mathrm{H}$ & $\mathrm{Pt}$ \\
\hline Setaria barbata (Lam.) Kunth & $\mathrm{T}$ & Asu \\
\hline $\begin{array}{l}\text { Setaria pumila (Poir.) Roem. \& } \\
\text { Schult. }\end{array}$ & $\mathrm{T}$ & $\mathrm{Pt}$ \\
\hline
\end{tabular}




\begin{tabular}{|c|c|c|c|}
\hline & $\begin{array}{l}\text { Sorghum bicolor (L.) Moench } \\
\text { Sporobolus helvolus (Trin.) } \\
\text { T.Durand \& Schinz }\end{array}$ & $\begin{array}{l}\mathrm{T} \\
\mathrm{P}\end{array}$ & $\begin{array}{l}\mathrm{Pt} \\
\text { Mas }\end{array}$ \\
\hline PORTULACACEAE & Portulaca oleracea $\mathrm{L}$. & $\mathrm{T}$ & $\mathrm{Pt}$ \\
\hline \multirow{10}{*}{ RUBIACEAE } & Diodia sarmentosa Swartz. & $\mathrm{T}$ & $\mathrm{Cp}$ \\
\hline & Diodia scandes Auct. & $\mathrm{T}$ & $\mathrm{Pt}$ \\
\hline & $\begin{array}{l}\text { Kohautia confusa (Hutch. \& } \\
\text { Dalziel) Bremek. }\end{array}$ & $\mathrm{T}$ & Asu \\
\hline & Mitracarpus villosus (Sw.) DC. & $\mathrm{T}$ & Af \\
\hline & Oldenlandia corymbosa $\mathrm{L}$. & $\mathrm{T}$ & $\mathrm{Pt}$ \\
\hline & Oldenlandia herbacea (L.) Roxb. & $\mathrm{T}$ & $\mathrm{Pt}$ \\
\hline & Spermacoce chaetocephala DC. & $\mathrm{T}$ & Af \\
\hline & Spermacoce radiata (DC.) Hiern & $\mathrm{T}$ & Af \\
\hline & Spermacoce ruelliae DC. & $\mathrm{T}$ & Af \\
\hline & Spermacoce stachydea DC. & $\mathrm{H}$ & As \\
\hline \multirow{2}{*}{ SOLANACEAE } & Physalis angulata $\mathrm{L}$. & $\mathrm{T}$ & $\mathrm{Pt}$ \\
\hline & Physalis micrantha Link. & $\mathrm{T}$ & Cosm \\
\hline TACCACEAE & Tacca involucrata Sch. Et Th & G & $\mathrm{Pt}$ \\
\hline URTICACEAE & Laportea aestuans (L.) Chew & $\mathrm{T}$ & $\mathrm{Pt}$ \\
\hline VERBENACEAE & Clerodendrum capitatum (Willd.) & $\mathrm{P}$ & Af \\
\hline \multirow{2}{*}{ VITACEAE } & $\begin{array}{l}\text { Ampelocissus pentaphylla (Guill. } \\
\text { \& Perr.) }\end{array}$ & $\mathrm{P}$ & Af \\
\hline & $\begin{array}{l}\text { Cayratia gracilis (Guill. et Perr.) } \\
\text { Suess }\end{array}$ & $\mathrm{T}$ & Af \\
\hline ZYGOPHYLLACEAE & Tribulus terrestris $\mathrm{L}$. & $\mathrm{T}$ & Cosm \\
\hline
\end{tabular}

Tableau 2 : Structure de la flore adventice des cultures de coton au Sénégal.

\begin{tabular}{lcccccc}
\hline \multirow{2}{*}{ Classe } & \multicolumn{2}{c}{ Famille } & \multicolumn{2}{c}{ Genre } & \multicolumn{2}{c}{ Espèce } \\
\cline { 2 - 7 } Dicotylédones & $\mathrm{N}$ & $\%$ & $\mathrm{~N}$ & $\%$ & $\mathrm{~N}$ & $\%$ \\
& 30 & 85,7 & 82 & 69,5 & 140 & 68,6 \\
Monocotylédones & 5 & 14,3 & 36 & 30,5 & 64 & 31,4 \\
\hline \multicolumn{1}{c}{ Total } & 35 & 100,0 & 118 & 100,0 & 204 & 100,0 \\
\hline
\end{tabular}


Tableau 3 : Richesse spécifique des familles et leur proportion.

\begin{tabular}{|c|c|c|}
\hline Famille & Nombre d'espèces & Proportion (\%) \\
\hline Fabaceae & 45 & 22,1 \\
\hline Poaceae & 41 & 20,1 \\
\hline Malvaceae & 19 & 9,3 \\
\hline Cyperaceae & 16 & 7,8 \\
\hline Convolvulaceae & 10 & 4,9 \\
\hline Asteraceae & 10 & 4,9 \\
\hline Rubiaceae & 10 & 4,9 \\
\hline Cucurbitaceae & 6 & 2,9 \\
\hline Amaranthaceae & 4 & 2,0 \\
\hline Combreataceae & 4 & 2,0 \\
\hline Euphorbiaceae & 4 & 2,0 \\
\hline Commelinaceae & 3 & 1,5 \\
\hline Lamiaceae & 3 & 1,5 \\
\hline Acantaceae & 2 & 1,0 \\
\hline Araceae & 2 & 1,0 \\
\hline Nyctaginaceae & 2 & 1,0 \\
\hline Pedaliaceae & 2 & 1,0 \\
\hline Solanaceae & 2 & 1,0 \\
\hline Vitaceae & 2 & 1,0 \\
\hline Aizoaceae & 2 & 1,0 \\
\hline Asparagaceae & 1 & 0,5 \\
\hline Capparaceae & 1 & 0,5 \\
\hline Caryophyllaceae & 1 & 0,5 \\
\hline Colchicaceae & 1 & 0,5 \\
\hline Icacinaceae & 1 & 0,5 \\
\hline Liliaceae & 1 & 0,5 \\
\hline Loganiaceae & 1 & 0,5 \\
\hline Onagraceae & 1 & 0,5 \\
\hline Phyllanthaceae & 1 & 0,5 \\
\hline Portulacaceae & 1 & 0,5 \\
\hline Scrophyllariaceae & 1 & 0,5 \\
\hline Taccaceae & 1 & 0,5 \\
\hline Urticaceae & 1 & 0,5 \\
\hline Verbenaceae & 1 & 0,5 \\
\hline Zygophyllaceae & 1 & 0,5 \\
\hline Total & 204 & 100,0 \\
\hline
\end{tabular}




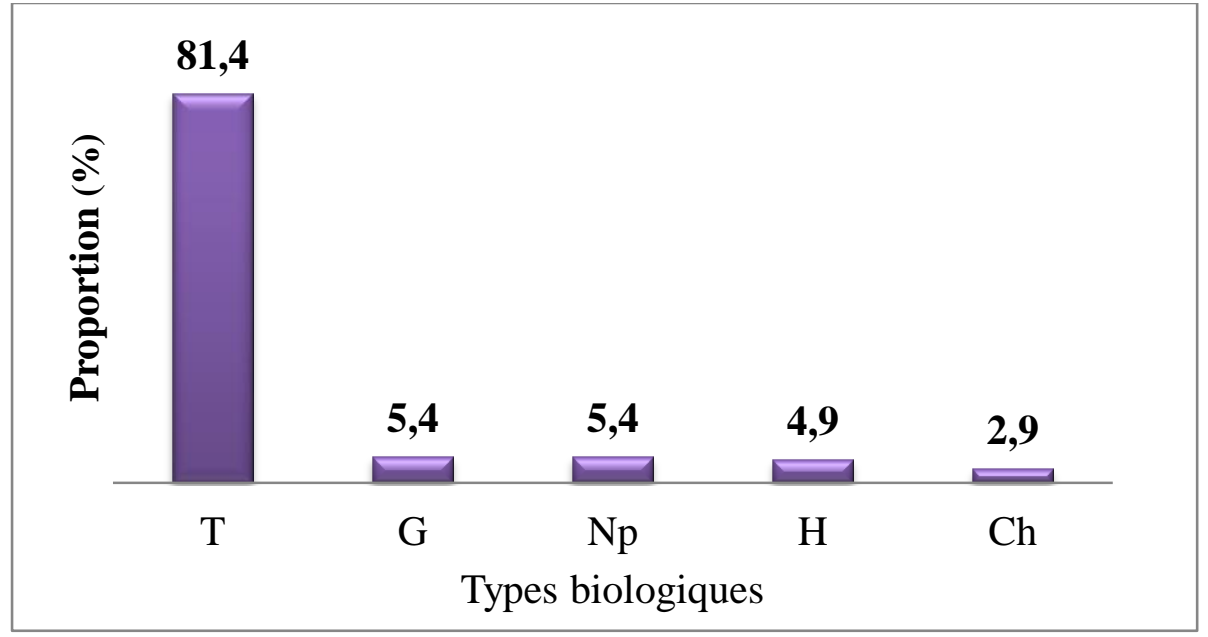

T= Thérophytes ; G= Géophytes ; Np= Nanophanérophytes ; Hémicryptophytes ; Ch= Chaméphytes

Figure 2: Types biologiques des espèces répertoriées.

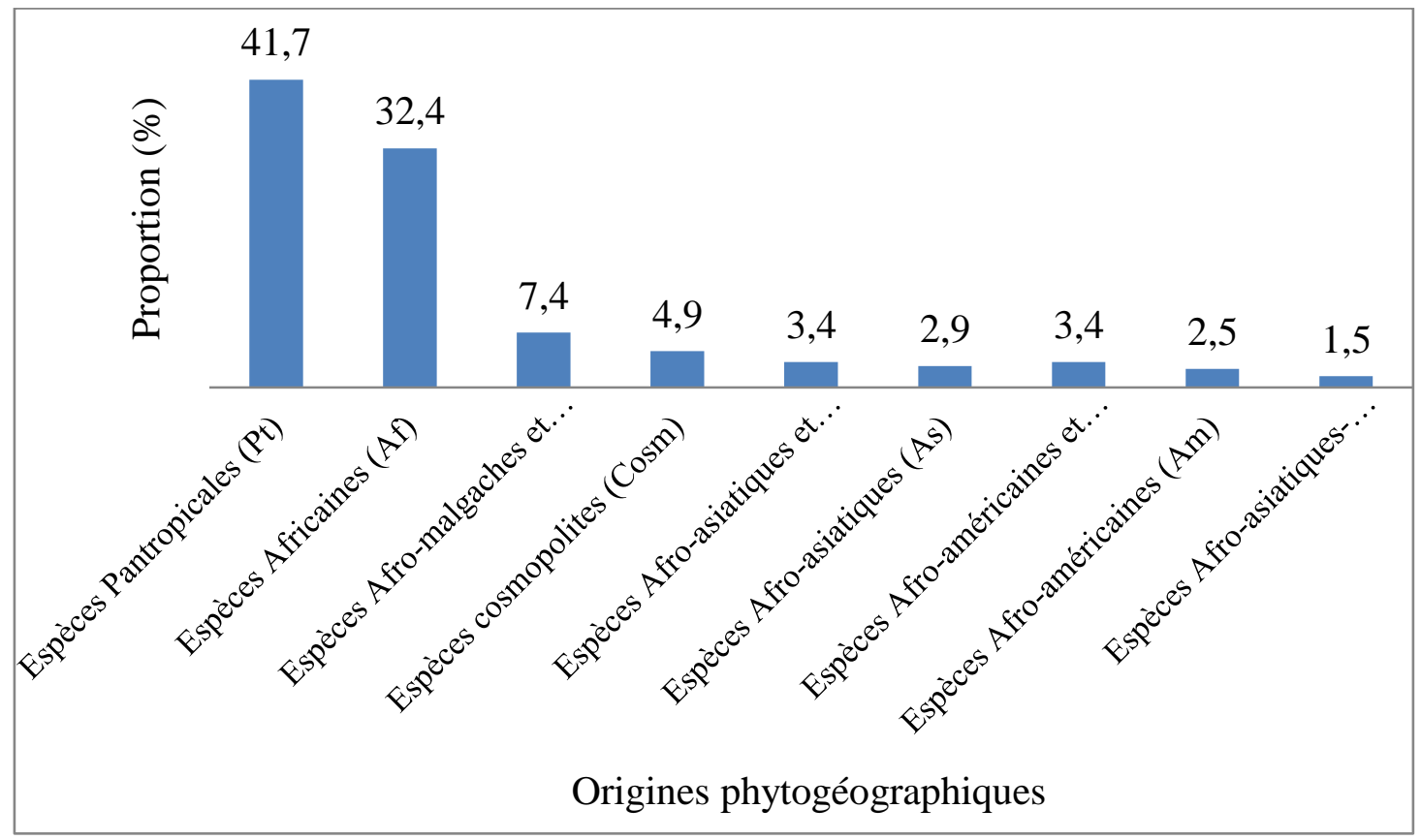

Figure 3: Répartition phytogéographique des espèces recensées.

Tableau 4: Indice de similitude entre les différents sites.

\begin{tabular}{lccc}
\hline Site & Zone humide & Zone intermédiaire & Zone sèche \\
\hline Zone humide & 100 & & \\
Zone intermédiaire & 75,25 & 100 & \\
Zone sèche & 65,25 & 63,3 & 100 \\
\hline
\end{tabular}




\section{DISCUSSION}

\section{Au plan de la structure floristique}

Les résultats obtenus à partir de cette étude d'inventaire montrent que la flore adventice dans le bassin cotonnier du Sénégal est très diversifiée comparée à celle du bassin arachidier du Sénégal. Dans cette zone du bassin arachidier du Sénégal, 125 espèces ont été répertoriées dans les cultures de mil et de l'arachide, 53 espèces dans les cultures de mil en association avec le Niébé et 128 espèces dans les cultures de maïs respectivement par Noba (2002), Mbaye (2013) et Bassène (2014). Cette diversité spécifique serait associée aux conditions climatiques favorables au développement de ces adventices. Cette plus forte diversité floristique dans le bassin cotonnier est liée entre autres au fait qu'il soit très pluvieux avec en moyenne près de $1000 \mathrm{~mm}$ par an. Noba (2002) et Mbaye (2013) ont montré que la pluviométrie est un facteur important pour la levée des adventices. En effet, avec une bonne pluviométrie, les semences des adventices germent puisque l'imbibition des téguments qui est nécessaire à la germination est assurée. Les familles des Fabaceae, Poaceae, Malvaceae et Cyperaceae présentent une importance relative en nombre d'espèces dans cette flore et à cet effet, elles sont responsables de l'enherbement des parcelles cotonnières au Sénégal. Ces quatre familles figurent parmi les dix (10) familles d'adventices considérées comme familles d'adventices mondiales. Il s'agit des Euphorbiaceae, Malvaceae, Asteraceae, Poaceae, Cyperaceae, Convolvulaceae, Fabaceae, Polygonaceae, Amaranthaceae et Solanaceae (Kouakou et al., 2016). Les familles des Fabaceae et Poaceae sont les plus représentées avec respectivement $22,3 \%$ et $19,8 \%$ des espèces de la flore inventoriée. Cette importance des Fabaceae s'expliquerait par l'utilisation de la classification APGIII qui y réunit trois (3) sous familles que sont: les Faboideae, les Mimoisoideae et les Caesalpinoidaea (Bassène, 2014). En ce qui concerne les Poaceae, leur importance s'expliquerait d'une part, par la présence de plusieurs parcelles de coton en zone de savane, les milieux de culture du coton sont des zones ouvertes si bien que la dispersion des espèces surtout herbacées $\mathrm{y}$ est très développée. Ces familles suscitées représentent les plus diversifiées en Afrique et dans le monde. En Algérie, les travaux de Kazi Tani et al. (2010) ont montré la prédominance des Poaceae et Fabaceae. Les résultats découlant de cette étude sont en conformité avec d'autres travaux antérieurs. En côte d'Ivoire, ceux de (Deat, 1976) ont montré que l'essentiel de la flore adventice dans les cultures cotonnières était constitué de Poaceae; même constat au Congo par (Miderho et al., 2017) dans les cultures de café. La supériorité numérique de ces familles au niveau de la flore adventice est également constatée par Guillerm et Maillet (1989) au sein de la flore des régions ouestméditerranéennes de l'Europe. La prédominance de ces familles est donc liée à leur adaptation aux différents milieux.

La flore adventice est fortement dominée par les Dicotylédones. Ces résultats corroborent ceux de Ka et al. (2017) trouvés dans les parcelles de sorgho en Haute Casamance. Ceci a été déjà observé au Sénégal, en Afrique et même dans le monde par plusieurs auteurs (Noba, 2002 ; Maye, 2013; Bassène, 2014) qui ont montré la prédominance de cette classe dans le sud du bassin arachidier; même situation au NordCameroun (Le bourgeois, 1993) et en zone périurbaine du district d'Abidjan (Côte d'Ivoire) (Kouamé et al., 2017). Cette dominance des Dicotylédones est aussi constatée en France dans les cultures annuelles de la côte d'Or (Dessaint et al., 2001).

\section{Sur le spectre biologique}

La flore adventice dans le bassin cotonnier du Sénégal est caractérisée par 5 types biologiques. Il s'agit des thérophytes, des géophytes, des hémicryptophytes, des chaméphytes des nanophanérophytes. Dans tous les travaux effectués dans les parcelles de cultures au Sénégal, la prédominance des 
thérophytes est remarquée (Noba, 2002; Mbaye, 2013 ; Bassène, 2014). Ce type biologique est le premier à se mettre en place dans les parcelles de cultures; Les travaux de préparation des parcelles tels que le labour et le sarclage favorisent le développement de ces espèces alors que les autres (Géophytes, Nanophanérophytes etc.) sont éliminées. Dans le bassin cotonnier du Sénégal, les thérophytes prédominent avec une proportion de plus de $82 \%$ des espèces recensées. De nombreux travaux en zone cotonnières ont révélé les mêmes observations avec une proportion de 93\% au Cameroun (Le bourgeois, 1993), plus de $77 \%$ au Benin (Ahanchede, 1995) et plus de $70 \%$ dans le secteur soudanais en Côte d'Ivoire (Kadio et al., 2004 ; Boraud et al., 2015 ; Ahonon et al., 2018). En outre, cette dynamique des thérophytes dans les parcelles cultivées est d'autant plus importante que les parcelles sont exposées au soleil puisque la majorité de ces espèces sont héliophiles. Les géophytes peuvent être considérées comme le type le plus dangereux car elles échappent au sarclage du fait de la présence de leurs bulbes en profondeur. Les Chaméphytes, les Nanophanérophytes, sont les moins bien représentées. Ces espèces restent rares dans les cultures et relèvent surtout du milieu naturel. Leur présence, dans ou à proximité des cultures relève le plus souvent d'une volonté de l'agriculteur (Touré et al., 2016).

\section{Sur le spectre chorologique}

L'importance des espèces pantropicales et Africaines de la flore adventice du bassin cotonnier du Sénégal $(75,1 \%)$ confirme bien les positions continentale et océanique des sites étudiés (Noba, 2002). Par ailleurs il faut noter une forte présence des espèces d'origine étrangère ce qui témoigne de la pénétration de beaucoup d'éléments (semences, fertilisants etc.) dans nos milieux de cultures (Bassène, 2014).

\section{Sur l'effet du site sur la flore}

Les trois localités (humide, intermédiaire et sèche) sont caractérisées par des flores adventices similaires. Ceci pourrait être expliqué entre autres par le fait qu'elles se trouvent dans des conditions édaphiques similaires alors que la composition floristique dépend également des conditions pédoclimatiques (Fried et al., 2008). Dans ces zones, le système de culture qui est un des facteurs les plus susceptibles de discriminer la flore adventice est le même dans tout le bassin cotonnier du Sénégal.

\section{Conclusion}

L'inventaire floristique effectué dans les parcelles cotonnières dans le bassin cotonnier du Sénégal a permis de recenser 204 espèces, dont 186 en zone humide, 103 en zone intermédiaire et 83 en zone sèche. Ces sites offrent une grande diversité floristique, avec 118 genres et 35 familles. Les Dicotylédones y sont les plus représentées avec $68,6 \%$ contre $31,4 \%$ pour les Monocotylédones. Les quatre familles (Fabaceae, Poaceae, Malvaceae, Cyperaceae) les mieux représentées comprennent à elles seules 121 espèces soit 59,3\% de l'effectif global. Les types biologiques dominants de cette flore adventice sont les thérophytes, avec $81,4 \%$. Sur le plan chorologique, cette flore est dominée par les espèces pantropicales $(41,7 \%)$ et africaines $(32,4 \%)$. Une analyse de la flore des trois sites que constitue le bassin cotonnier du Sénégal laisse apparaître une homogénéité floristique avec des coefficients de similitude de 75,$25 ; 65,25$ et $63,3 \%$.

\section{CONFLIT D'INTERETS}

Les auteurs déclarent qu'ils n'ont aucun conflit d'intérêts.

\section{CONTRIBUTIONS DES AUTEURS}

ND a contribué à la rédaction du protocole, a collecté les données, a contribué à l'analyse et au traitement des données et a rédigé le manuscrit. $\mathrm{CB}$ et $\mathrm{BD}$ ont contribué à la rédaction du manuscrit. MG a contribué à la collecte des données et à la rédaction du manuscrit. KN et MSM ont 
supervisé les travaux et ont contribué à la rédaction du manuscrit.

\section{REMERCIEMENTS}

Les auteurs remercient les autorités de l'Université de Dakar et du Département de Biologie Végétale, sans qui ce travail n'aurait pu être mené à son terme, ainsi que l'ISRA pour la mise en œuvre des moyens matériels et financiers qui ont permis l'aboutissement de ce travail.

\section{REFERENCES}

Ahanchede A. 1995. Mauvaises herbes des cultures pluviales au nord-est du Benin. Faculté des sciences Agronomiques. Cotonou, Benin. 8 p.

Ahonon BA, Traoré H, Ipou Ipou J. 2018. Mauvaises herbes majeures de la culture de Haricot (Phaseolus vulgaris L.) dans la région de Moronou au Centre Est de la Côte d'Ivoire. Int. J. Biol. Chem. Sci., 12(1): 310-321. DOI : https://dx.doi.org/10.4314/ijbcs.v12il.25

Bassène C. 2014. La flore des systèmes pastoraux de la basse Casamance (Sénégal) : Cas de la communauté rurale de Mlomp. Int. J. Biol. Chem. Sci., 8(5): 2258-2273.

DOI : https://dx.doi.org/10.4314/ijbcs.v8i5.25

Berhaut J. 1967. Flore du Sénégal (2ème édn). Clairafrique : Dakar; p257.

Boraud NKM, Kouamé Kra F, Kla D. 2015. Impacts des pratiques de gestion des adventices sur le rendement du riz au centre de la Côte d'Ivoire. Int. J. Biol. Chem. Sci., 9(3): 1220-1228. DOI : https://dx.doi.org/10.4331/ijbcs.v9i3.7

Boudjedjiou L. 2010. Etude de la flore adventice de la région de Jijel, Mémoire de Magister. Faculté des sciences, Département de Biologie, Université Ferhat Abbas-Sétif. 155 p.

Déat M. 1976. Les adventices en cultures cotonnières en Côte d'Ivoire. Cot. Fib. Trop., 31(4) : 419-427.

Dessaint F, Chadoeuf R, Barralis G. 2001. Diversité des communautés de mauvaises herbes des cultures annuelles de Côte-d'Or (France) - Biotechnol. Agron. Soc. Environ., 5(2) : 91-98.

Diaw M. 2010. Valorisation des coproduits de la graine de coton exempte de glandes à gossypol en production de poulets au Sénégal ; Thèse de Doctorat. Académie Universitaire Wallonie-Europe. Université de Liège. p168

Fried G, Chauvel B, Reboud X. 2008. Evolution de la flore adventice des champs cultivés au cours des dernières décennies vers la sélection des groups d'espèces répondant aux systèmes de cultures. LNPV, station d'entomologie, Sys Agro Bâtiment 18, Montpellier, France. Innovation agronomique 2008, p15-26.

Gounot M. 1969 . Méthode d'Etude Quantitative de la Végétation. Masson: Paris ; $314 \mathrm{p}$.

Guillerm JL, Maillet J. 1989. Westerm Mediterranean countries of Europe. In Biology and Ecology of Weeds, Holzner W, Numata M (eds.). W. Junk Pub., The Hague; p 227-243.

Ka SL, Guéye M, Mbaye MS, Bamba B, Ly MO, Diouf N, Noba K. 2017. Systematic composition, life forms chorology of fallow lands in Eastern Senegal and Casamance, Senegal. Int. J. Biol. Chem. Sci., 11(6): 2573-2586. DOI : https://dx.doi.org/10.4314/ijbcs.v11i6.2

Kadio GA, Ipou Ipou J, Toure Y . 2004. La flore des adventices des Cultures Cotonnières de la Région du Worodougou, Au Nord-Ouest De La Cote D'ivoire. Laboratoire de Botanique. UFR Biosciences, Université de Cocody. Agronomie Africaine, 16 (1) : 1-14.

Kazi Tani Ch, Le Bourgeois T, Munoz F. 2010. Aspects floristiques des adventices $\mathrm{du}$ domaine phytogéographique oranais (Nord- Ouest Algérien) et persistance d'espèces rares et endémiques. $F l$ Médit., 20 : 29-46.

Kouakou JN, Kouame FK, Ipou Ipou J, Gue A. 2016. Importance relative des mauvaises herbes de la culture du maïs dans le département de M'Bahiakro. 
Journal of Innovation and Applied Studies.

Kouamé AS, Bakayoko GA, Kouamé KF, Ipou IJ, N'guessan KE. 2017. Flore adventice des cultures vivrières de la zone périurbaine du district d'Abidjan (Côte d'Ivoire). J. Appl. Biosci., 118 : 11744-11753.

Le Bourgeois T. 1993. Les mauvaises herbes dans la rotation cotonnière au Nord Cameroun (Afrique) - Amplitude d'habitat et degré d'infestation - Cycle de développement. Thèse de Doctorat, USTL, Montpellier, France, 241p.

Lebrun JP, Stork AL. 1991 et 1997. Enumération des Plantes à Fleurs d'Afrique Tropicale. Conservatoire et Jardin Botanique de Genève. 4 volumes.

Maillet J. 1981. Evolution de la flore adventice dans le Montpelliérais sous la pression des techniques culturales. Thèse Doctorat, Montpellier, p200.

Mbaye MS. 2013. Association mil [Pennisetum glaucum (L.) R. Br.] et niébé [(Vigna unguiculata (L.) Walp.] : arrangement spatiotemporel des cultures, structure, dynamique et concurrence de la flore adventice et proposition d'un itinéraire technique. Thèse de Doctorat d'Etat de Biologie Végétale. FST, UCAD, Dakar. 236p.

Miderho CC, Birimwiragi FM, Kadorho AS, Bisimwa BH, Shamamba DB, Safari IM, Nkuba BG, Masamba WJ. 2017. Inventaire Floristique des Mauvaises Herbes dans une Caféiculture en Pure dans le Territoire de Kabare, DR Congo. Département de phytotechnie, Faculté de
Sciences Agronomiques, Université Catholique de Bukavu (UCB). p13.

Ndong JB. 1995. L'évolution de la pluviométrie au Sénégal et les incidences de la sécheresse récente sur l'environnement. Rev. Géogr. Lyon, 70(3) : 193-198.

Ndour A, Romain L, Gourlot JP, Ba KS, Dieng A, Clouvel P. 2017. Changement climatique et production cotonnière au Sénégal : concevoir autrement les stratégies de diffusion des variétés. SODEFITEX. Dakar (Sénégal). Biotechnol. Agron. Soc. Environ., 21(1) : 22-35.

Noba K. 2002. La flore adventice dans le sud du Bassin arachidier (Sénégal) : structure dynamique et impact sur la production du mil et de l'arachide. Thèse de Doctorat d'Etat de Biologie Végétale. FST, UCAD, Dakar. p128.

Raunkiaer C. 1934. The Life Froms of Plants and Statistical Plants Geography. Clarendron Press: Oxford; 623p.

Thiombiano A, Schimdt M, Dressler S, Ouédrago A, Hahn K, Zizka G. 2012. Catalogue des plantes vasculaires du Burkina Faso. Mémoire de botanique systématique, Volume 65, Genève, 391p.

Touré A, Adou LMD, Kouamé FK, Ipou JI. 2016. Dynamique d'infestation de la forêt classée de Sanaimbo par les adventices à partir des agroécosystèmes environnants. Tropicultura, 34(4) : 361374. 